\title{
Adecuación de la Calidad Microbiológica de Desechos de Lagunas de Estabilización por la Aplicación de Ozono
}

\author{
Luci Sartori, Luiz A. Daniel y Harry E. Schulz \\ Universidad de São Paulo, Escuela de Ingeniería de São Carlos, Departamento de Hidráulica y \\ Saneamiento, Av. Trabalhador São-carlense N400, 13566-590 São Carlos, SP-Brasil \\ (e-mail: sartori@sc.usp.br, Idaniel@sc.usp.br, heschulz@sc.usp.br)
}

\begin{abstract}
Resumen
Se evalúa la acción del ozono en la desinfección de desechos secundarios de alcantarillado sanitario, usando como microorganismos indicadores Escherichia coli, colifagos y Clostridium perfringens. Los desechos secundarios utilizados provenían de las plantas de tratamiento de las ciudades de Lins y Araraquara en Brasil. Los resultados mostraron que valores de concentración de ozono en el agua 29, 54, 82, 164 y $246 \mathrm{mg}$ min /L para el desecho de Lins y de 25, 95, 142, 147, 189, 220 y $294 \mathrm{mg}$ min /L de Araraquara, fueron adecuados, de acuerdo con los valores establecidos por la Organización Mundial de la Salud, en lo referente a la concentración de E. coli, para su uso en el regadío de cultivos. Se concluye que la aplicación de ozono, para regular la calidad microbiológica de los desechos de alcantarillado es adecuada. Sin embargo, la eficiencia de desinfección es afectada por el tratamiento previo que se realiza a los desechos.
\end{abstract}

Palabras clave: alcantarillado, desinfección, microorganismos, ozono, calidad microbiológica

\section{Microbiological Quality Adequation of Effluents from Secondary Lagoons by the Application of Ozone}

\begin{abstract}
The disinfection of secondary effluents with ozone using Escherichia coli, coliphages and Clostridium perfringens as indicator microorganisms was evaluated. The secondary effluents of Lins Wastewater Treatment Plant and Araraquara in Brazil, were used. The results showed that values of ozone-inwater concentration of 29, 54, 82, 164 and $246 \mathrm{mg}$ min /L for Lins effluent and 25, 95, 142, 147, 189, 220 and $294 \mathrm{mg} \mathrm{min} \mathrm{/L} \mathrm{for} \mathrm{Araraquara} \mathrm{effluent} \mathrm{were} \mathrm{adequate,} \mathrm{according} \mathrm{to} \mathrm{the} \mathrm{standards} \mathrm{of} \mathrm{the}$ World Health Organization for unrestricted irrigation, considering only $E$. coli concentration. It is concluded that the application of ozone to regulate the microbiological quality of the wastewaters is adequate. However, the efficiency of disinfection is affected by the previous treatment given to the wastes.
\end{abstract}

Keywords: wastewater, disinfection, microorganisms, ozone, microbiological quality 


\section{INTRODUCCIÓN}

El aumento de la demanda por recursos hídricos, agravados por la polución y contaminación de manantiales, tienen como resultado la necesidad de tratamiento y uso del alcantarillado sanitario como suplemento a las necesidades de agua en actividades agrícolas, industriales y demás actividades urbanas (WPCF, 1989). Cuando se considera el tipo de demanda, en el ámbito global, se observa que entre $63-73 \%$ del agua se destina al regadío de cultivos. En Brasil, a pesar de no existir una tradición de regadío, se constata que aproximadamente $60 \%$ del agua es destinada al regadío de mono-cultivos. Para la práctica segura del regadío con alcantarillado sanitario tratado, es necesario asegurar que el desecho contemple las normas y patrones sugeridos por la OMS (1989), y aunque el tratamiento reduzca la concentración de microorganismos patogénicos, es difícil asegurar la completa eliminación de estos.

La desinfección puede suministrar agua con calidad deseada para actividades como, por ejemplo, regadío y recarga de acuíferos. A pesar del cloro ser el desinfectante de uso mas difundido, estudios han demostrado que este, en presencia de la materia orgánica forma subproductos indeseables con propiedades carcinogénicas y mutagénicas (Rathbun, 1997; Fawell, 2000). Como una forma de minimizar la formación de esos subproductos, y proporcionar seguridad en relación a la inactivación de microorganismos patogénicos, otros desinfectantes están siendo usados en sustitución al cloro.

El ozono inactiva eficientemente amplia variedad de microorganismos, siendo superior al cloro al considerarse quistes de Giardia y ooquistes de Cryptosporidium (Kim et al., 2002; Paraskeva y Graham, 2002, Larson y Mariñas, 2003).

Aunque el ozono sea de uso amplio y de eficiencia comprobada en tratamiento de agua de abastecimiento, es poco usado para desinfección de aguas residuales (Paraskeva y Graham, 2002; Xu et al, 2002) la cual se remonta a la década de 1970 (Buffle et al., 2006a y 2006b)

Actualmente la aplicación del ozono en aguas residuales, ha recibido mayor atención con el descubrimiento, de su capacidad en degradar eficientemente ciertas clases de compuestos farmacéuticos, generando productos oxidados inócuos (Buffle et al., 2006a, Buffle et al., 2006b).

El proceso de ozonización de aguas residuales, a pesar de la inversión en investigación, es poco conocido. Los estudios enfocan con mayor atención aspectos empíricos, como la optimización de variables operacionales teniendo como control, la cuantificación de microorganismos sobrevivientes. Con eso, es difícil prever resultados solamente en dosis aplicadas o dosis consumidas (Paraskeva y Graham, 2002).

El ozono, puede, además de la desinfección, remover gusto y olor, aumentar la eficiencia de la coagulación y filtración, remover color, prevenir el crecimiento de algas y, oxidar hierro y manganeso (USEPA, 1999).

En esta investigación se estudió la desinfección de desechos secundarios con ozono utilizando como variable de control la inactivación de Escherichia coli, colifagos y Clostridium perfringens.

\section{MATERIAL Y MÉTODOS}

La unidad de desinfección en escala de laboratorio (Fig. 1), estaba constituida de un cilindro de oxígeno comercial $(98,6 \%)$, cámara de refrigeración, generador de ozono con capacidad para la producción de $8 \mathrm{~g} \mathrm{O}_{3} / \mathrm{h}$, frasco colector de gas residual que contiene solución de $\mathrm{KI}$ a $2 \%$ y cámara de contacto de sección cilíndrica en PVC, con diámetro interno de $100 \mathrm{~mm}$ y altura total de 2,0 m. Los desechos de las plantas de tratamiento de alcantarillado (PTA), fueron cargados en el tope de la columna y después de la desinfección, eran colectados en la base de la propia columna. La operación era hecha en partes.

Los desechos secundarios provenían de la PTA-Lins, cuyo tratamiento consiste en laguna anaerobia seguida de laguna facultativa, y de la PTA-Araraquara, en la cual el tratamiento estaba constituido 
de laguna aireada, seguida de laguna de sedimentación (ambas estaciones se ubican en São PauloBrasil). Los desechos eran colectados en las salidas de las lagunas facultativa (PTA-Lins) y de sedimentación (PTA-Araraquara), almacenados en recipientes de plástico y transportados para la sede de la Universidad de São Paulo (São Carlos, São Paulo), mantenidos refrigerados a $4 \pm 0.2^{\circ} \mathrm{C}$, por periodo máximo de 24 horas.

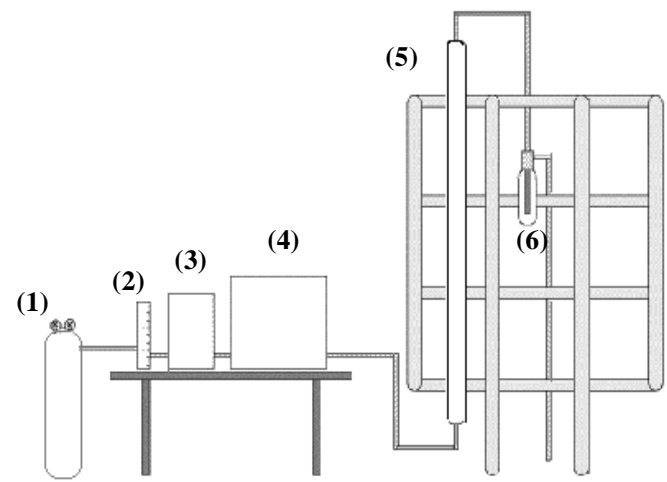

(1) Cilindro de oxígeno

(2) Rotámetro

(3) Cámara de refrigeración

(4) Generador de ozono

(5) Cámara de contacto

(6) Frasco colector de gas residual

Fig. 1: Esquema de la unidad de desinfección en escala de laboratorio (Bilotta, 2000)

Fueron realizadas 12 corridas de ensayos (numeradas del I a XII), cada corrida se hacía en triplicado. Para el desecho de la PTA-Lins, la concentración de ozono consumido fue de $6 \pm 0.3$, $10 \pm 0.65$ y $18 \pm 1.08 \mathrm{mg} / \mathrm{L}$, para tiempos de contacto de 10,20 y $30 \mathrm{~min}$., respectivamente. El desecho de la PTA-Araraquara, fue desinfectado empleándose tiempos de contacto de 10, 15 y 20 min. Para cada tiempo de contacto la dosis de ozono consumido fue $10 \pm 0.70,20 \pm 0.8$ y $30 \pm 1.5 \mathrm{mg} / \mathrm{L}$, respectivamente.

Los análisis de concentración residual de ozono (método 4500-O $\mathrm{O}_{3}$ ) alcalinidad (método 2320) y sólidos - sólidos suspendidos totales (SST), sólidos suspendidos fijos (SSF), sólidos totales (ST) (método 2540) fueron realizados de acuerdo con los Métodos Estándar para la Evaluación de Aguas y Tratamiento de Aguas, (APHA, 1998).

Para la cuantificación de $E$. coli se utilizó el método de Colilert $®$. La bacteria esporulada $C$. perfringens, fue cuantificada por la técnica de los tubos múltiples, procedimiento descrito por CETESB (L5.213, 1993). Los colifagos fueron determinados por el procedimiento descrito por CETESB $(L 5.225,1990)$ y adaptado por el Departamento de Microbiología del Instituto de Ciencias Biomédicas de la Universidad de São Paulo, utilizándose la cepa hospedera de E. coli CIP 55.30.

\section{RESULTADOS Y DISCUSIÓN}

\section{Desecho de la PTA-Lins}

Las corridas I, II y III corresponden a los tiempos de contacto de 10, 20 y 30 minutos y concentración de ozono consumido de $6 \pm 0.3,10 \pm 0.65$ y $18 \pm 1.08 \mathrm{mg} / \mathrm{L}$, respectivamente. Las corridas IV, $\mathrm{V}$ y $\mathrm{VI}$ corresponden a las réplicas de esos ensayos. Las características físicas, químicas y biológicas del desecho de la laguna facultativa se presentan en la Tabla 1.

El desecho de la laguna facultativa, debido a la presencia de algas, presentó alta alcalinidad, con una media de $313 \pm 10.1 \mathrm{mg} \mathrm{CaCO} / \mathrm{L}$, y sólidos suspendidos totales de $104 \pm 36.3 \mathrm{mg} / \mathrm{L}$. El número más probable por $100 \mathrm{~mL}(\mathrm{NMP} / 100 \mathrm{~mL})$ de E. coli varió de $10^{4}$ a $10^{7}$ y el NMP/100 mL de $C$. perfringes varió de $10^{5}$ a $10^{6}$. Los colifagos variaron de 500 a 9500 UFP/100 mL (unidad formadora de placa por $100 \mathrm{~mL}$ ).

La inactivación de E. coli, de acuerdo a lo mostrado en la Fig. 2, fue mayor en los tiempos de contacto de 10 y 30 min., para todas las dosis de ozono consumido. La menor eficiencia, ocurrió en 
el tiempo de contacto próximo a los 20 minutos. La concentración de ozono tiene menor influencia en la eficiencia de desinfección, pues al fijarse el tiempo de contacto y variar la concentración de ozono, resulta en pequeño aumento de eficiencia con el aumento de dosis.

Tabla 1: Características físico-químicas y microbiológicas del desecho de la PTA-Lins antes de la desinfección

\begin{tabular}{|l|c|c|c|c|c|c|}
\hline Variable & $\begin{array}{c}\text { Corrida } \\
\text { I }\end{array}$ & $\begin{array}{c}\text { Corrida } \\
\text { II }\end{array}$ & $\begin{array}{c}\text { Corrida } \\
\text { III }\end{array}$ & $\begin{array}{c}\text { Corrida } \\
\text { IV }\end{array}$ & $\begin{array}{c}\text { Corrida } \\
\text { V }\end{array}$ & $\begin{array}{c}\text { Corrida } \\
\text { VI }\end{array}$ \\
\hline E.coli (NMP/100mL) & $10^{5}$ & $10^{4}$ & $10^{7}$ & $10^{5}$ & $10^{5}$ & $10^{5}$ \\
\hline Colifagos (UFP/100mL) & 500 & 1500 & 1550 & 9500 & 1500 & 950 \\
\hline C.perfringens (NMP/100mL) & $10^{5}$ & $10^{5}$ & $10^{5}$ & $10^{6}$ & $10^{6}$ & $10^{5}$ \\
\hline pH & 7.9 & 7.7 & 7.6 & 7.4 & 7.6 & 7.7 \\
\hline Alcalinidad (mg CaCO $3 / \mathrm{L})$ & 316 & 316 & 302 & 324 & 300 & 322 \\
\hline SST (mg/L) & 167 & 58 & 90 & 110 & 111 & 89 \\
\hline SSF (mg/L) & 9.9 & 3.4 & 14 & 7.9 & 11 & 12 \\
\hline SSV (mg/L) & 155 & 55 & 75 & 102 & 99 & 76 \\
\hline ST (mg/L) & 730 & 700 & 869 & 659 & 675 & 725 \\
\hline
\end{tabular}

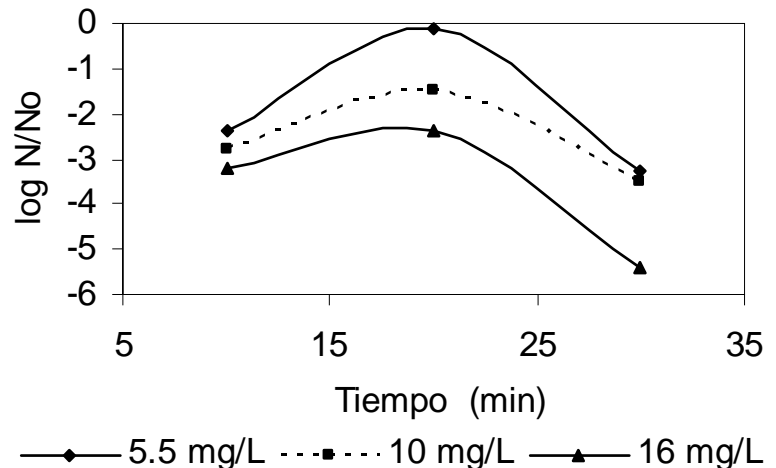

Corridas I, II y III

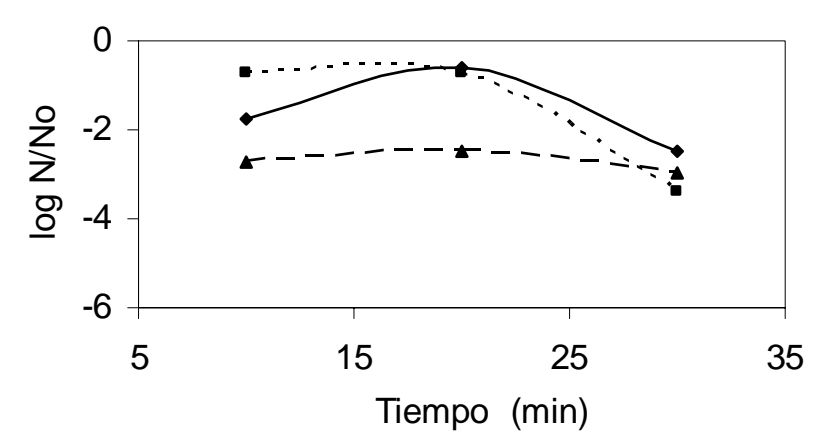

$5.5 \mathrm{mg} / \mathrm{L} \ldots \ldots 10 \mathrm{mg} / \mathrm{L}-\rightarrow--16 \mathrm{mg} / \mathrm{L}$

Corridas IV, $\vee$ y VI

Fig. 2: Inactivación de E.coli con ozono - efluente PTA Lins-SP

Tomando como base las variables físico-químicas analizadas, no es posible identificar las causas de la menor eficiencia para el tiempo de contacto de 20 minutos, incluso aumentándose la dosis de ozono. Se observa que el desecho de la laguna facultativa, contiene concentración elevada de sólidos suspendidos totales, en consecuencia de la gran cantidad de algas, y alcalinidad elevada, la cual inhibe la formación de radicales libres por la descomposición del ozono, lo que debería resultar en mayor eficiencia de desinfección. Sin embargo, los sólidos suspendidos envuelven a los microorganismos protegiéndolos de la acción del ozono.

Los ensayos fueron realizados en partes, con aplicación continua de ozono en el transcurso del tiempo de contacto. Para las concentraciones de 6 y $10 \mathrm{mg} / \mathrm{L}$, todo el ozono suministrado era consumido, independientemente del tiempo de contacto. Para la concentración de $18 \mathrm{mg} / \mathrm{L}$, se verificaba la existencia de ozono residual en el tope de la columna. De cualquier forma, la masa de ozono utilizada en cada experimento es conocida (aumentando con la concentración y con el tiempo de contacto).

En principio, no habiendo otro tipo de competencia con reacciones químicas, se espera que la cantidad total de microorganismos eliminados, debe ser proporcional a la masa total consumida, 0 en otras palabras, la inactivación debe crecer tanto con el tiempo como con la concentración. Ese punto de vista es defendible y pueden ser propuestas bases matemáticas que permitan cuantificarlo. De manera inmediata, es posible argumentar que, manteniendo las condiciones de toxicidad (concentración de ozono), la tasa de mortalidad (número de organismos muertos por unidad de 
tiempo) es proporcional al número de organismos presentes (mientras más elementos existen, más mueren). En otras palabras:

$\frac{d N}{d t}=-\lambda N$

$N$ : número de organismos, $\lambda$ : constante de decaimiento de la población; $t$ : tiempo.

Se trata del modelo más simple de decaimiento de la población, que, integrado, produce:

$\ln \left(\frac{N}{N_{0}}\right)=-\lambda t$

$N_{0}$ :número de elementos de la población considerada en el tiempo inicial.

Siendo aplicable ese simple modelo, la Fig. 2, debería mostrar un decrecimiento lineal de $\log \left(N / N_{0}\right)$ con el tiempo, lo que no se verifica. Considerando, sin embargo, modelos más elaborados de crecimiento poblacional, en los cuales la interacción entre miembros de una misma especie es considerada, las expresiones matemáticas son más complejas, como es el caso de la ecuación logística del crecimiento poblacional, que puede ser escrita en la forma:

$$
\frac{d N}{d t}=-\lambda_{M} N(1-N)
$$

Donde $N$ está vinculado al número de elementos y $\lambda_{M}$ involucra las tasas de mortalidad y de natalidad, o, en otras palabras, es un parámetro que considera la interacción entre los organismos vivos y el medio, siendo este último quién impone condiciones más o menos favorables al crecimiento. May (1976) y Feigenbaum (1978), mostraron que, para representar una población que varía de forma discreta (no es posible tener una fracción de organismo vivo, pero sí apenas un organismo entero vivo), la ecuación (3) debe asumir la forma:

$$
\Pi_{t+1}=-\theta_{M} \Pi_{t}\left(1-\Pi_{t}\right)
$$

$\Pi$, también está vinculado al número de elementos de la población (después de algunos algebrismos, la población pasa a ser multiplicada por algunas constantes, pero el significado del análisis permanece inalterado), mientras $\theta_{M}$ tiene el mismo significado de $\lambda_{M}$, también considerando algunas constantes. $t$ representa el tiempo en un instante genérico y $t+1$ representa un momento posterior, considerando un intervalo de tiempo finito en relación a $t$. May(1976) y Feigenbaum (1978), demostraron que la ecuación (4), puede producir diferentes tipos de comportamiento a lo largo del tempo, en función del parámetro $\theta_{M}$. Los siguientes comportamientos son posibles, y están descritos detalladamente por los autores citados anteriormente:

(1) Decrecimiento continuo, en los moldes de la ecuación (2).

(2) Crecimiento continúo hasta la saturación (máxima población para las condiciones vigentes).

(3) Decrecimiento-Crecimiento oscilatorio, o sea, la población puede variar periódicamente con el tiempo, siguiendo un comportamiento tipo senoidal o más complejo, recordando un patrón de superposición de funciones senoidales con diferentes períodos. 
(4) Decrecimiento-crecimiento caótico, o sea, los valores de población no presentan ninguna periodicidad con el tiempo y no se repiten, pudiendo alcanzar valores muy altos o muy bajos de forma aleatoria.

Todos esos comportamientos son observados en poblaciones reales de microorganismos, incluso en situaciones de trabajo bastante próximas. Por tanto, considerar la "tasa de crecimiento" en conjunto con la "tasa de mortalidad", como forma de interpretar la respuesta del organismo vivo al impulso externo, parece ser adecuado para justificar comportamientos orgánicos que escapan al patrón físico-químico usualmente utilizado (respuestas proporcionales a los parámetros de control como tiempo y concentración, por ejemplo). Se puede decir, de forma simplificada, que la respuesta es consecuencia de una "competencia", de la propia especie por las mejores condiciones de supervivencia (o de la mejor adecuación de cada organismo a las condiciones externas).

De esta forma, en el contexto del presente trabajo, las respuestas temporales que presentan comportamientos no monótonos, repetidamente observados, pueden ser consecuencia de la competencia interna, ya que, como muestran los comportamientos (3) y (4) mencionados, puede haber aumento y disminución periódica o aleatoria en el número de microorganismos. Además se debe recordar que puede haber una competencia externa, entre la oxidación de la materia orgánica y la inactivación de microorganismos. En otras palabras, el ozono disponible para la inactivación puede estar siendo utilizado, tanto por reacciones químicas como para la inactivación. Considerando que la tasa de transferencia de ozono para cada porción que lo consume (materia orgánica o microorganismos) puede variar con el tiempo de contacto (10, 20 o 30 min., en las Fig. 2 y 3 ). Una respuesta definitiva sobre la causa exacta de los valles observados en las Fig. 2 y 3 , aún no es posible, pero los indicios muestran que las diferentes formas de competencia parecen ser la causa general.

La inactivación de Clostridium perfringens (Fig. 3), mantuvo un comportamiento semejante al de $E$. coli, presentando menor eficiencia de inactivación en el tiempo de contacto de 20 minutos. La inactivación de los colifagos, fue mayor mientras mayor fue el tiempo de contacto y la dosis de ozono, no ocurriendo la reducción de eficiencia para el tiempo de contacto de 20 minutos. Para la dosis consumida de ozono entre 16 y $18 \mathrm{mg} / \mathrm{L}$, hubo $100 \%$ de inactivación para los tiempos de contacto 10, 20 y 30 minutos (Fig. 4).
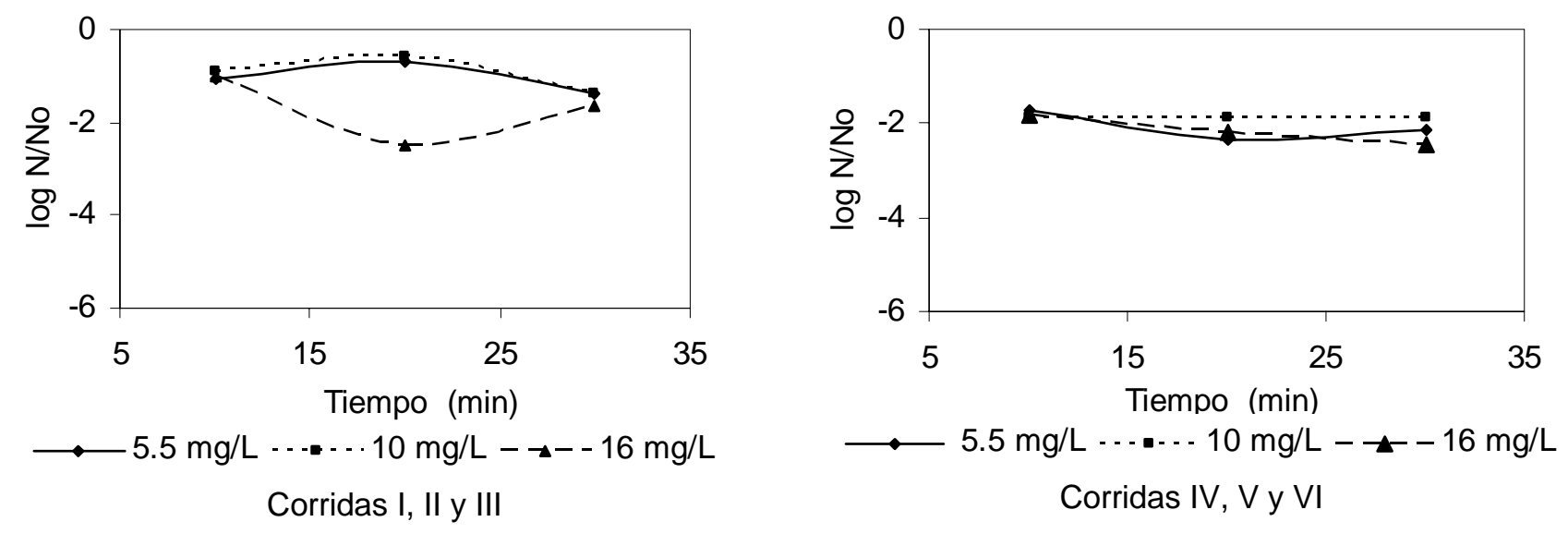

Fig. 3: Inactivación de C. perfringens con ozono - efluente PTA Lins-SP

\section{Desecho de la PTA-Araraquara}

Los ensayos fueron realizados empleando tiempos de contacto de 10, 15 y 20 minutos y concentraciones de ozono consumido $10 \pm 0.70,20 \pm 0.80$ y $30 \pm 1.50 \mathrm{mg} / \mathrm{L}$ para cada tiempo de contacto, que corresponde a las corridas VII, VIII y IX, respectivamente. Las corridas X, XI y XII corresponden a las réplicas de esos ensayos. Las características físicas, químicas y microbiológicas del desecho de la PTA-Araraquara están presentadas en la Tabla 2. 


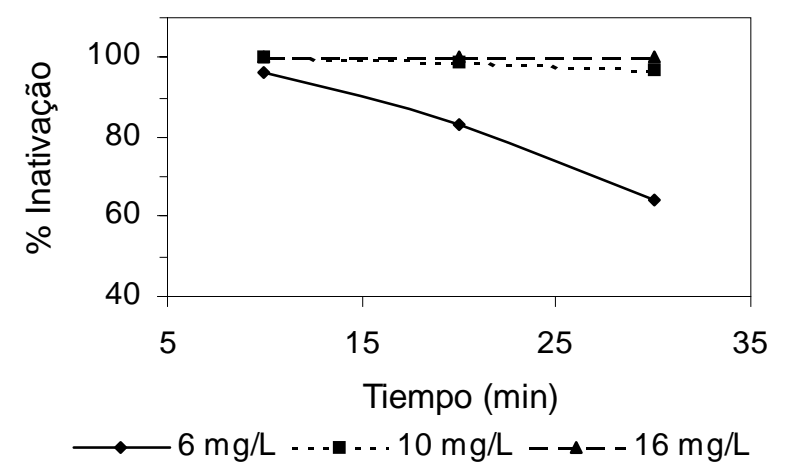

Corridas I, II y III

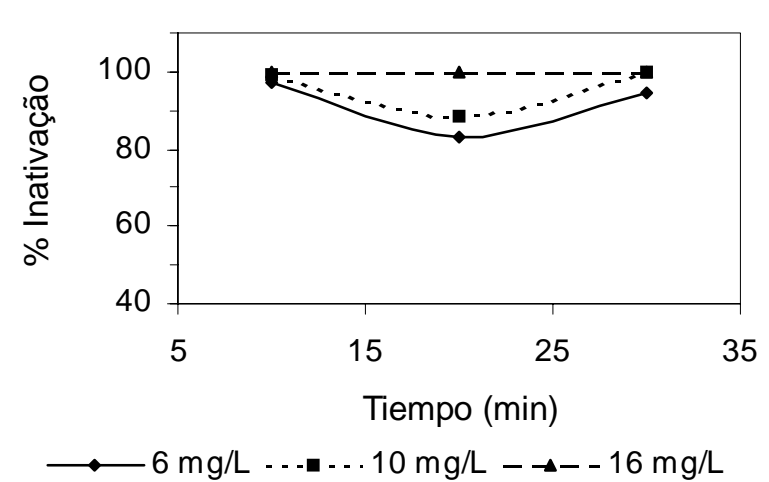

Corridas IV, $\mathrm{V}$ y $\mathrm{VI}$

Fig. 4: Inactivación de colífagos con ozono - efluente PTA Lins-SP

Tabla 2: Características físico-químicas y microbiológicas del desecho de la PTA-Araraquara antes de la desinfección

\begin{tabular}{|l|c|c|c|c|c|c|}
\hline Parámetros & $\begin{array}{c}\text { Corrida } \\
\text { VII }\end{array}$ & $\begin{array}{c}\text { Corrida } \\
\text { VIII }\end{array}$ & $\begin{array}{c}\text { Corrida } \\
\text { IX }\end{array}$ & $\begin{array}{c}\text { Corrida } \\
\text { X }\end{array}$ & $\begin{array}{c}\text { Corrida } \\
\text { XI }\end{array}$ & $\begin{array}{c}\text { Corrida } \\
\text { XII }\end{array}$ \\
\hline E.coli $(\mathrm{NMP} / 100 \mathrm{~mL})$ & $10^{4}$ & $10^{4}$ & $10^{5}$ & $10^{4}$ & $10^{5}$ & $10^{4}$ \\
\hline Colifagos (UFP/100mL) & 2700 & 3700 & 4000 & 3450 & 3500 & 4000 \\
\hline $\begin{array}{l}\text { C.perfringens } \\
(\mathrm{NMP} / 100 m \mathrm{~m})\end{array}$ & $10^{5}$ & $10^{5}$ & $10^{4}$ & $10^{5}$ & $10^{5}$ & $10^{4}$ \\
\hline $\mathrm{pH}$ & 7.0 & 7.1 & 7.0 & 6.8 & 6.5 & 7.0 \\
\hline Alcalinidad (mg CaCO $/ \mathrm{L})$ & 95 & 115 & 81 & 50 & 47 & 81 \\
\hline $\mathrm{SST}(\mathrm{mg} / \mathrm{L})$ & 33 & 30 & 43 & 35 & 46 & 43 \\
\hline $\mathrm{SSF}(\mathrm{mg} / \mathrm{L})$ & 2.5 & 5 & 8 & 1.7 & 6 & 8 \\
\hline $\mathrm{SSV}(\mathrm{mg} / \mathrm{L})$ & 31 & 25 & 35 & 34 & 40 & 35 \\
\hline $\mathrm{ST}(\mathrm{mg} / \mathrm{L})$ & 400 & 374 & 400 & 600 & 406 & 400 \\
\hline
\end{tabular}

El desecho de la PTA-Araraquara, en comparación al desecho de la PTA-Lins, presentó menor alcalinidad (promedio de $78.17 \pm 26.16 \mathrm{mg} \mathrm{CaCO}_{3} / \mathrm{L}$ ) y menor concentración de sólidos suspendidos totales (promedio de $38.33 \pm 6.50 \mathrm{mg} / \mathrm{L}$ ), lo que modificó el comportamiento de la inactivación de los micro-organismos indicadores (Fig. 5, 6 y 7). Para la E. coli (Fig. 5), se observa que las mayores eficiencias de inactivación son alcanzadas, cuando se aplica una concentración de ozono consumido por sobre $10 \mathrm{mg} / \mathrm{L}$, y tiempo de contacto mayor a 10 minutos. Por el contrario, de los resultados observados con el desecho de Lins-SP, para el tiempo de contacto de 20 minutos, no se observó reducción en la eficiencia de inactivación.

La mayor eficiencia de inactivación de C. perfringens fue obtenida para la condición de tiempo de contacto mayor a 16 minutos y concentración de ozono consumido entre 16 y $24 \mathrm{mg} / \mathrm{L}$, no ocurriendo una disminución de inactivación para el tiempo de contacto de 20 minutos. Los $C$. perfringens fueron resistentes a la acción del ozono, pues la inactivación permaneció inferior a 1.5 (Fig. 6) en la mayoría de las condiciones experimentales (Liberti et al., 2000).

Los colifagos fueron inactivados $100 \%$ con un tiempo de contacto de 20 min., y de dosis de ozono consumido comprendido entre 10 a $30 \mathrm{mg} / \mathrm{L}$ (Fig. 7).

Hay divergencias cuanto a la necesidad de manutención de residual de ozono para efectivarse la desinfección. Gehr et al. (2003) concluyeron que mayor inactivación de E. coli y de G. muris ocurrió cuando había ozono residual, al contrario de lo que ocurrió cuando el ozono transferido fue rápidamente consumido. Sin embargo, Xu et al. (2002), observaron inactivación de coliformes fecales, cuando la dosis de ozono transferido fue próxima a la demanda de ozono, con obtención de 1 a 3 log de inactivación. 


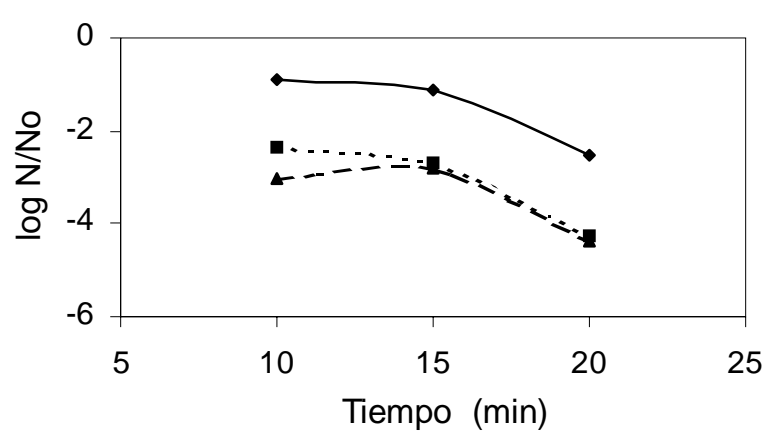

$\longrightarrow 10 \mathrm{mg} / \mathrm{L} \ldots \ldots 18 \mathrm{mg} / \mathrm{L}-\rightarrow--30 \mathrm{mg} / \mathrm{L}$

Carreras VII, VII y IX

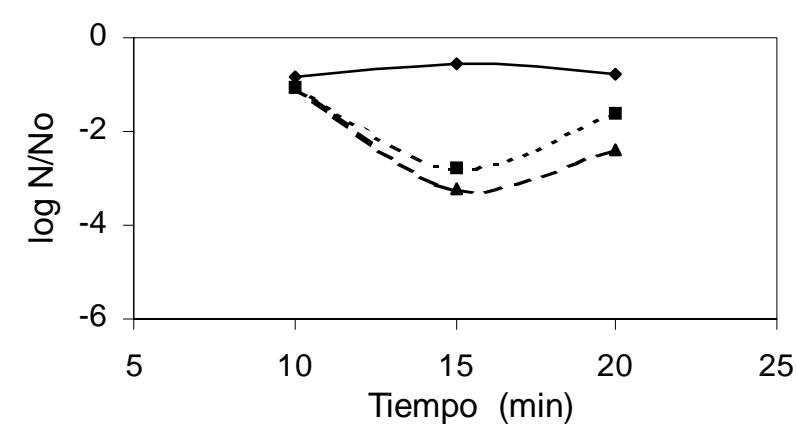

$\longrightarrow-10 \mathrm{mg} / \mathrm{L} \ldots \ldots 19 \mathrm{mg} / \mathrm{L}-\rightarrow-30 \mathrm{mg} / \mathrm{L}$

Carreras X, XI y XII

Fig. 5: Inactivación de E.coli con ozono - efluente PTA-Araraquara-SP

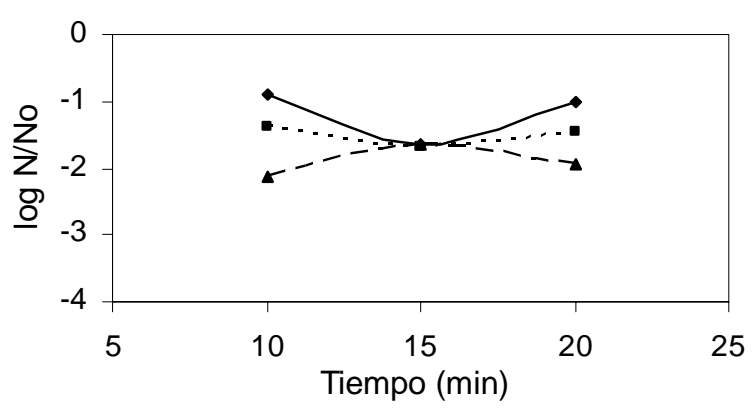

$\longrightarrow 10 \mathrm{mg} / \mathrm{L} \ldots . . .18 \mathrm{mg} / \mathrm{L}-\rightarrow--30 \mathrm{mg} / \mathrm{L}$

Carreras VII, VII y IX

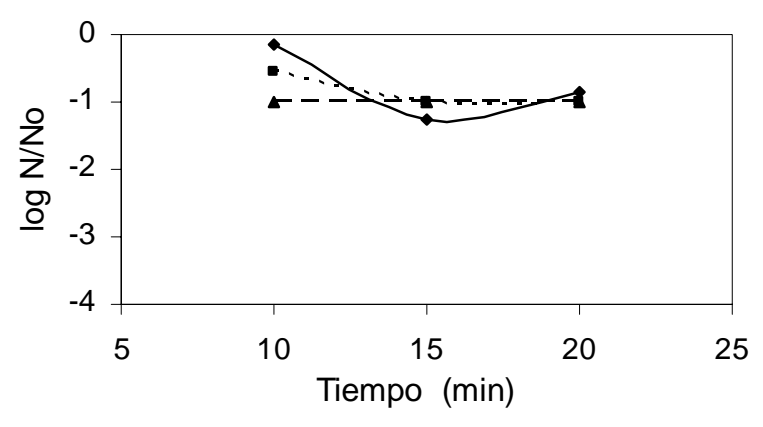

$\longrightarrow 10 \mathrm{mg} / \mathrm{L} \ldots \ldots 19 \mathrm{mg} / \mathrm{L}-\rightarrow-30 \mathrm{mg} / \mathrm{L}$

Carreras $\mathrm{X}, \mathrm{XI}$ y XII

Fig. 6: Inactivación de C. perfringens con ozono - efluente PTA Araraquara-SP

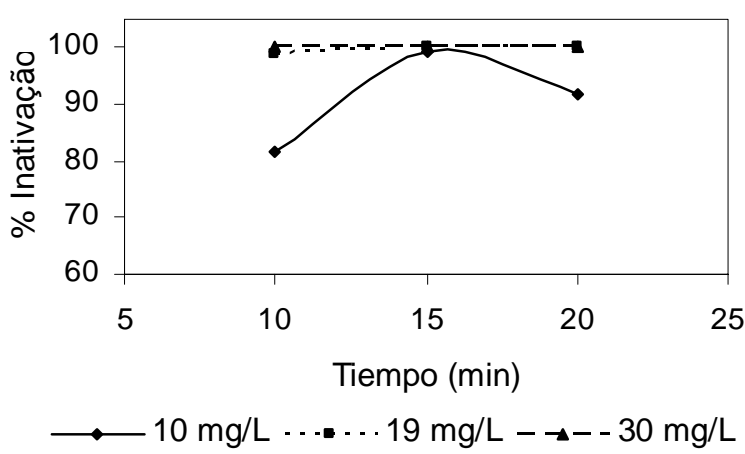

Carreras VII, VII y IX

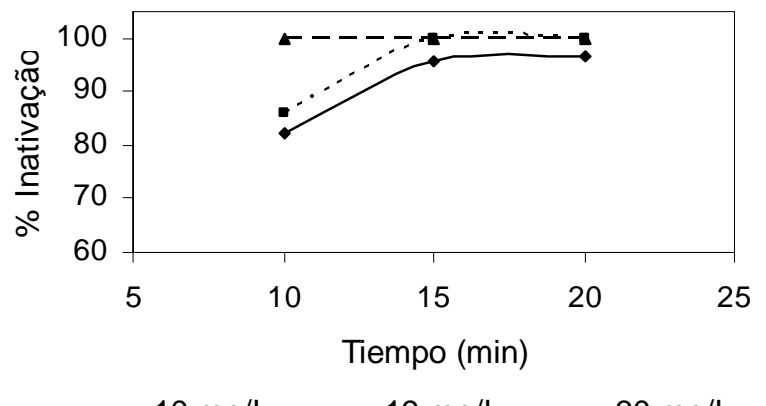

Carreras X, XI y XII

Fig. 7: Inativactivación de colífagos con ozono - efluente PTA Araraquara-SP

Rakness et al. (2005), presentaron diferentes métodos de cálculo de CT para desinfección con ozono. La dificultad está en definir cuál es la concentración residual de ozono, la cual varía con el tiempo. Por eso, el uso del concepto de CT para la desinfección de aguas residuales con ozono, no es recomendado por Xu et al. (2002), debido al consumo rápido de ozono.

La mayoría de los estudios fueron realizados para el agua de abastecimiento. Existe poca información para aguas residuales, para el cual, el consumo de ozono es alto desde el inicio de la aplicación (Paraskeva y Graham, 2002).

La masa de ozono aplicada fue rápidamente consumida y no fue observada limitación de 
transferencia de masa, comprobado por la concentración residual de ozono en cantidad despreciable, y pequeña cantidad que escapó en el gas residual. Ante a estas dificultades, se optó por calcular CT considerando la dosis de ozono consumido (Rakness et al., 2005), que resulta en concentración de microorganismos menor o igual a $1000 \mathrm{NMP} / 100 \mathrm{~mL}$, usando el procedimiento de Wu y Doan (2005) (Tabla 3).

Tabla 3: Valores de CT para E. coli considerando concentraciones de microorganismos después de la desinfección menor o igual a 1000 UFC/100 mL

\begin{tabular}{|c|c|c|c|c|c|c|c|}
\hline PTA-Lins & \multicolumn{3}{|c|}{ CT } & PTA-Araraquara & \multicolumn{3}{c|}{ CT } \\
\hline $\begin{array}{c}\text { Ozono consumido } \\
(\mathrm{mg} / \mathrm{L})\end{array}$ & \multicolumn{2}{|c|}{ Tiempo de contacto $(\mathrm{min})$} & Ozono consumido & \multicolumn{2}{c|}{ Tiempo de contacto (min) } \\
\cline { 2 - 6 } & 10 & 20 & 30 & $(\mathrm{mg} / \mathrm{L})$ & 10 & 15 & 20 \\
\hline 5.8 & 29 & & & 10.0 & & & 25 \\
\hline 10.8 & 54 & & & 18.9 & 95 & 142 & 189 \\
\hline 16.4 & 82 & 164 & 246 & 29.4 & 147 & 220 & 294 \\
\hline
\end{tabular}

\section{CONCLUSIONES}

La resistencia de los microorganismos indicadores a la acción desinfectante del ozono, se presentó en el siguiente orden, tanto para el desecho de la PTA-Lins (laguna anaerobia y laguna facultativa) como para el desecho de la PTA-Araraquara (laguna aireada y laguna de sedimentación): colifagos (E. coli hospedera CIP 55.30), E. coli y C. perfringens.

El proceso de tratamiento de alcantarillado sanitario interfirió en la eficiencia de desinfección con ozono.

El desecho de la PTA-Lins presentó, una región de menor eficiencia para tiempo de contacto de 20 min para $E$. coli y $C$. perfringens, lo que no posibilita establecer relación general para CT. La inactivación de E. coli, C. perfringens y colifagos para el desecho de la PTA-Araraquara, con relación directa, entre dosis de ozono y tiempo de contacto, no posibilita establecer una relación entre eficiencia de desinfección y CT.

Los $C$. perfringens fueron resistentes al ozono, manteniendo concentración sobreviviente superior a $1 \times 10^{3}$ organismos $/ 100 \mathrm{~mL}$. Debido a esta mayor resistencia, pueden ser utilizados como indicadores de protozoarios.

\section{AGRADECIMIENTOS}

Los autores agradecen a la FAPESP por los recursos concedidos (proceso 00/08253-5).

\section{REFERENCIAS}

APHA, AWWA, WEF; Standard Methods for the Examination of Water and Wastewater, $20^{a}$ edición, Washington (1998).

Bilotta, P.; Estudo Comparativo da Ação do Ozônio e Radiação UV na Desinfecção de Esgoto Sanitário, Tesis de Magíster, São Carlos: Escola de Engenharia de São Carlos, Universidade de São Paulo (2000).

Buffle, M.O. y otros cuatro autores; Measurement of the Initial Phase of Ozone Decomposition in Water and Wastewater by Means of a Continuous Quench-Flow System: Application to Disinfection and Pharmaceutical Oxidation, Water Research: 40(9), 1884-1894 (2006a).

Buffle, M.O. y otros cuatro autores; Ozonation and Advanced Oxidation of Wastewater: Effect of $\mathrm{O}_{3}$, Dose, $\mathrm{pH}$, DOM and HO $\mathrm{HO}^{\circ}$-Scavengers on Ozone Decomposition ad HO${ }^{\bullet}$ Generation, Ozone Science and Engineering: 28(4), 247-259 (2006b) 
Fawell, J.; Risk Assessment Case Study-Chloroform and Related Substances, Food and Chemical and Toxicology: 38, 91-95 (2000).

Feigenbaum, M.J.; Quantitative universality for a class of nonlinear transformations Journal of Statistical Physics: 19(1) 25-52 (1978)

Gehr, R., M. Wagner, P. Veerasubramanian y P. Payment; Disinfection efficiency of peracetic acid, UV and ozone after enhanced primary treatment of municipal wastewater Water Research: 37(19), 4573-4586 (2003)

Kim, J.H., R. Tomiak. y B.J Mariñas; Inactivation of Cryptosporidium oocysts in a pilot-scale ozone bubble -diffuser contactor. I: model development J. Environ. Engineering: 128(6), 514-520 (2002)

L5 213; Determinação do número mais provável de clostridio sulfido-redutores (Clostridium perfringens): método de ensaio, Cetesb, 28p., São Paulo, Brasil, (1993).

L5 225; Determinação de Colifagos em amostras de água: método de ensaio, Cetesb, 26p.,São Paulo, Brasil, (1990).

Larson, M.A. y B.J. Mariñas; Inactivation of Bacillus subtilis spores with ozone and monochloramine, Water Research: 37(4), 833-844 (2003).

Liberti, L., M. Notamicola y A. Lopez; Advanced Treatment for Municipal Wastewater Reuse in Agriculture.III - Ozone Disinfection, Ozone Science and Engineering: 22, 151-156 (2000).

May, R.M.; Simple mathematical models with very complicated dynamics. Nature: 261(5560),.459467 (1976).

OMS; Guidelines for the use of wastewater in agriculture and aquaculture, Geneva: World Health Organization (1989).

Paraskeva, P. y N.D. Graham; Ozonation of Municipal Wastewater Effluents, Water Environment Research: 74(6), 569-581 (2002).

Rakness, K L. y otros cuatro autores; Cryptosporidium log-inactivation with ozone using effluent $C T_{10}$, geometric mean $C T_{10}$, extended integrated $C T_{10}$ and extended CSTR calculations, Ozone Science Engineering: 27(5), 335-350 (2005)

Rathbun, R.E.; Effect of reaction time on the formation of disinfection byproducts, Chemosphere: 34(12), 2699-2713 (1997).

USEPA; U.S. Environmental Protection Agency, Microbial and Disinfection Byproduct Rules Simultaneous Compliance Guidance Manual, Washington EPA-815/R-99-015 (1999).

WPCF; Water Pollution Control Federation, Water Reuse. Manual of practice: SM-3. USA (1989)

$\mathrm{Wu}$, J. y $\mathrm{H}$. Doan; Disinfection of recycled red-meat-processing wastewater by ozone, Journal of Chemical Technology and Biotechnology: 80, 828-833 (2005)

$\mathrm{Xu}$, P. y otros cuatro autores; Wastewater disinfection by ozone: main parameters for process design Water Research: 36(4), 1043-1055 (2002). 